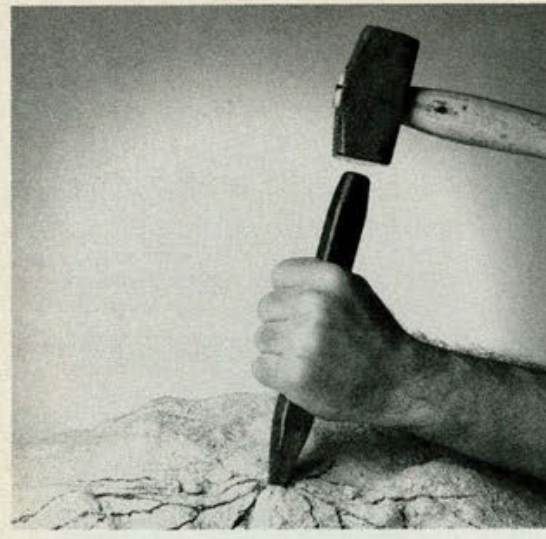

\section{Break through the congestion.}

\section{GIDIFED Capsules \\ (pseudoephedrine $\mathrm{HCl} 120 \mathrm{mg}$ and guaifenesin $250 \mathrm{mg}$ )}

GUAIFED-PD ${ }^{\circledR}$ Capsules (pseudoephedrine $\mathrm{HCl} 60 \mathrm{mg}$ and guaifenesin $300 \mathrm{mg}$ )

\section{Brief Summary}

CONTRAINDICATIONS: This product is contraindicated in patients with a known hypersensitivity to any of its ingredients. Also contraindicated in patients with severe hypertension, severe coronary artery disease and patients on MAO inhibitor therapy. Should not be used during pregnancy or in nursing mothers.

Considerable caution should be exercised in patients with hypertension, diabetes melitus, ischemic heart disease, hyperthyroidism, increased intraocular pressure and prostatic hypertrophy. The elderly ( 60 years or older) are more likely to exhibit adverse reactions. At dosages higher than the recommended dose, nervousness, dizziness or sleeplessness may occur.

PRECAUTIONS: General: Caution should be exercised in patients with high blood pressure, heart disease, diabetes or thyroid disease and in patients who exhibit difficulty in urination due to enlargement of the prostate gland. Check with a physician if symptoms do not improve within 7 days or

Drug Interactions: Do not take this product if you are presently taking a prescription drug for high blood pressure or depression, without first consulting a physician. MAO inhibitors and beta adrenergic blockers may increase the effect of sympathomimetics. Sympathomimetics may reduce the antihypertensive effects of methyldopa, mecamylamine reserpine and veratrum alkaloids. Pseudoephedrine hydrochloride may increase the possibility of cardiac arrhythmias in patients presently taking digitalis glycosides.

Pregnancy: Pregnancy Category B. It has been shown that pseudoephedrine hydrochloride can cause reduced average weight, length, and rate of skeletal ossification in the animal fetus.

Nursing Mothers: Pseudoephedrine is excreted in breast milk; use by nursing mother is not recommended because of the higher than usual risk of side effects from sympathomimetic amines for infants, especially newborn and prema-
ture infants.

Geriatrics: Pseudoephedrine should be used with caution in the elderly because they may be more sensitive to the effects of the sympathomimetics.

WARNINGS: Do not take this product for persistent or chronic cough such as occurs with smoking, asthma, or emphysema, or where cough is accompanied by excessive secretions except under the advice and supervision of a physician. This medication should be taken a few hours prior to bedtime to minimize the possibility of sleepless ness. Take this medication with a glass of water after each dose, to help loosen mucus in the lungs.

ADVERSE REACTIONS: Adverse reactions include nausea, cardiac palpitations, increased irritability or excite, shortness of breath and/or troubled breathing

DOSAGE AND ADMINISTRATION: GUAIFED ${ }^{*} \mathrm{CAP}^{*}$ SULES Adults and children over 12 years of age: 1 capsule every 12 hours.

GUAIFED-PD CAPSULES Adults and children over 12 years of age: 1 or 2 capsules every 12 hours. Children 6 to 12 years of age: 1 capsule every 12 hours.

CAUTION: FEDERAL (U.S.A.) LAW PROHIBITS DISPENSING WITHOUT A PRESCRIPTION.

\title{
federal update
}

\section{From the FDA}

\section{AZT indication extended to use in children}

The Food and Drug Administration announced that it has granted permission for distribution of zidovudine (Retrovir) (AZT) for use in treating children younger than 13 years of age who have acquired immunodeficiency syndrome (AIDS) or who have symptoms of advanced infecton with the human immunodeficiency virus (HIV).

Under a treatment investigational new drug (IND) program, AZT will be distributed free of charge to children with AIDS or in advanced stages of HIV infection. The Burroughs Wellcome Company of Research Triangle Park, NC, is sponsoring the program. The National Institute of Allergy and Infectious Diseases (NIAID), a component of the National Institutes of Health (NIH), is providing medical and technical assistance.

This treatment IND classification is based on clinical data that indicate that the drug may prolong survival and relieve severe AIDS symptoms in children, as it does in adults. These data were obtained through clinical trials sponsored by Burroughs Wellcome and conducted by researchers at the National Cancer Institute and through NIAID's AIDS Clinical Trials
Group network. The pediatric trials, begun in 1986, have involved more than 200 children.

Under the treatment IND protocol, physicians caring for children aged 3 months to 12 years who have symptoms of advanced HIV infection will be eligible to receive the drug at no cost. A recently approved strawberry-flavored syrup form of AZT will be used because it is more easily swallowed by children and the doses can be more easily adjusted to their body size than can the capsule form of the drug.

Zidovudine has been approved since March 1987. It is indicated for the treatment of patients aged 13 and older who have severe symptoms of HIV infection. Label indications do not restrict physicians from prescribing AZT for other patient populations, including children younger than 13 years. However, many physicians and hospitals have been reluctant to use AZT for children because it is not labeled for this use, and because of concerns that the drug's known side effects in adults might be even more severe in children. Clinical data now indicate that children receiving AZT experience side effects similar to those occurring in adults.

Although AZT is the only drug that has been shown to be effective in prolonging the (continued on page 19) 


\section{Easy $m g$-for-mg switch to convenient, once-daily dosing}

- Over $90 \%$ of angina patients controlled on nifedipine capsules were easily switched to and controlled on PROCARDIA XL Extended Release Tablets at the nearest equivalent total daily dose; others needed dosage adjustment ${ }^{2}$

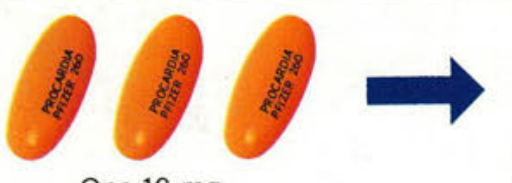

One 10-mg nifedipine capsule t.i.d.

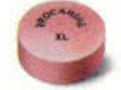

One 30-mg PROCARDIA XL Extended Release Tablet q.d.

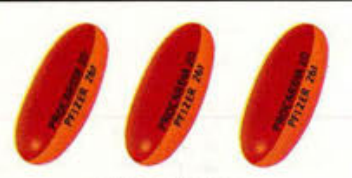

One 20-mg nifedipine capsule t.i.d. d.

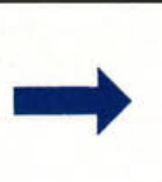
PROCARDIA XL Tablet q.d.

\section{Switch from Nifedipine Capsules to PROCARDIA XL Extended Release Tablets}

\section{Capsules}

$30-40 \mathrm{mg} /$ day in divided doses $\rightarrow 30 \mathrm{mg}$ once a day $50-70 \mathrm{mg} /$ day in divided doses $\rightarrow 60 \mathrm{mg}$ once a day $80-100 \mathrm{mg} /$ day in divided doses $\rightarrow 90 \mathrm{mg}$ once a day

- Subsequent dosage adjustments may be necessary and should be initiated as clinically warranted

- The total daily dose can be given with multiple tablets once a day, such as two 60-mg PROCARDIA XL Extended Release Tablets to equal $120 \mathrm{mg}$ total daily dose

- Experience with doses $>90 \mathrm{mg}$ in patients with angina is limited; therefore, doses $>90 \mathrm{mg}$ should be used with caution and only when clinically warranted

Call 1-800-NOW-RX-XL for additional information on switching to once-a-day PROCARDIA XL Extended Release Tablets.

\footnotetext{
References:

1. Vetrovec GW, Parker VE. Cole S, et al: Nifedipine gastrointestinal therapeutic system in stable angina pectoris: Results of a multicenter open-label cro

2. Data on file. Medical Department, Pfizer Laboratories, Pfizer Inc, New York.
}

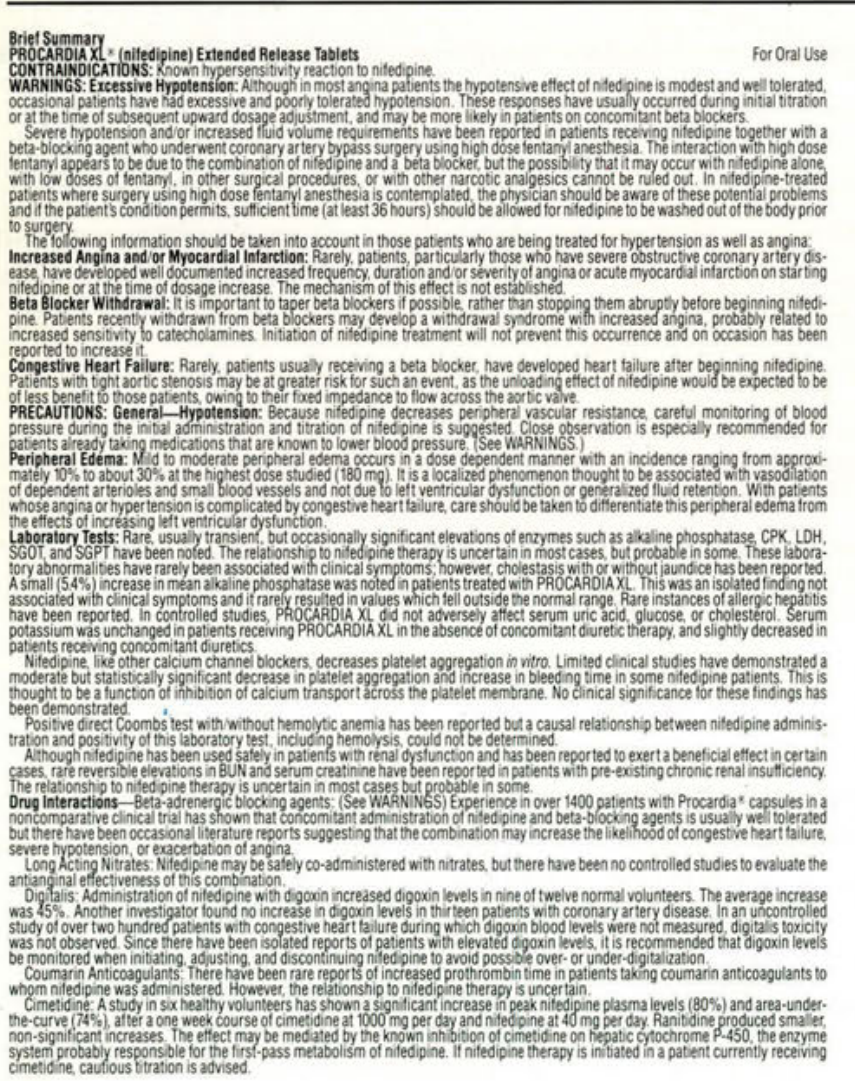

Carcinogenesis, Mutagenesis, Impairment of fertility: Nifedipine was administered orally to rats, for two vears and was not shown to
be carchogenic, When oven to rats proor to mating. nifedipine caused reduced tertility at a dose approximately 30 times the maximum

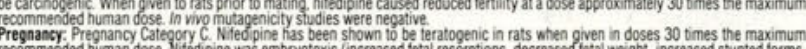

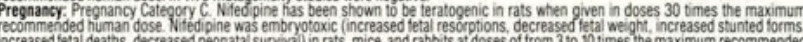

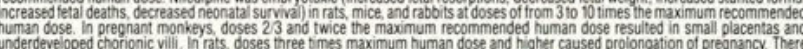

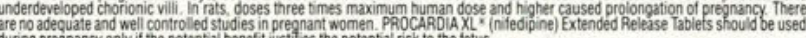

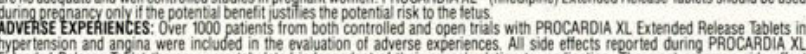

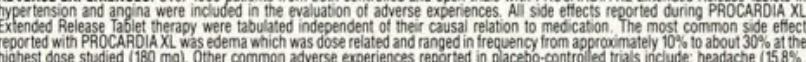

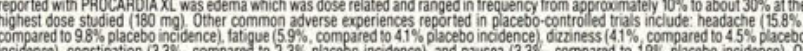

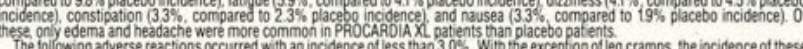

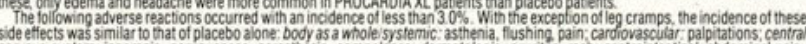

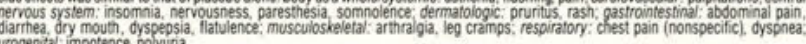

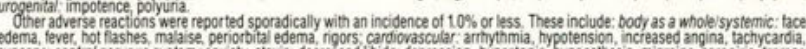

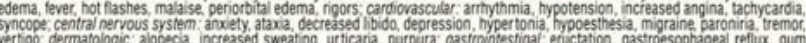

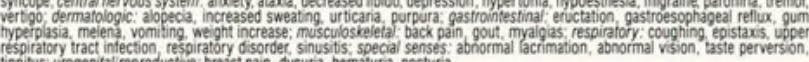

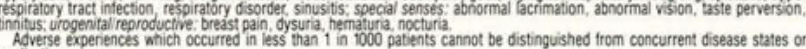
medications In multiple-dose U.S. and foreign controlled studies with nitedipine capsules in which adverse reactions were reported spontaneously.

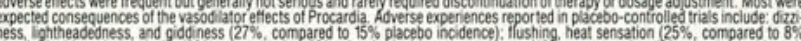

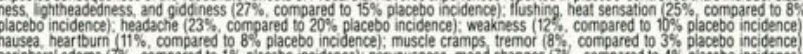

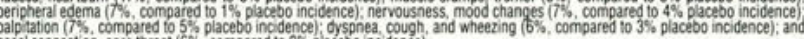
There Is also a large uncontrolled experience in over 2000 patients in the United States. Nost of the patients had vasospastic of

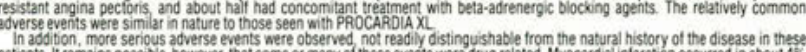

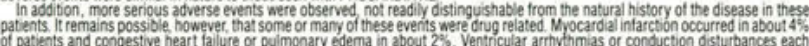

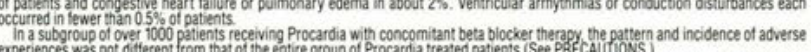

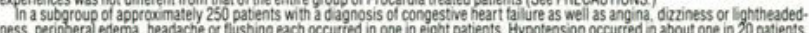

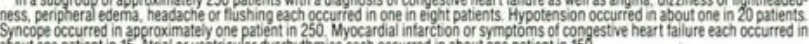


lives of AIDS patients, it is also known to have significant side effects. The drug can inhibit erythrocyte production, which may result in severe anemia requiring blood transfusions. Zidovudine can also reduce the leukocyte counts to the level where the drug's use has to be discontinued, to avoid infections.

Using data accrued from the pediatric clinical trials of AZT, the treatment IND has been designed to minimize the risks of these side effects. $\mathrm{Pa}$ tients enrolled in this treatment IND program will be carefully monitored for any adverse reactions.

Physicians interested in enrolling patients in the treatment IND protocol may call the Burroughs Wellcome tollfree number at 1-800-829PEDS from 8 AM to 7 PM eastern time. The company will immediately begin processing applications from physicians for their pediatric patients.

\section{Agency approves liquid ibuprofen for children}

The FDA has approved a liquid formulation of ibuprofen (Children's Advil Suspension, Whitehall Laboratories, New York). It is to be administered to reduce fever and treat the symptoms of juvenile arthritis in children younger than 12 years. In clinical trials, 5 $\mathrm{mg} / \mathrm{kg}$ doses administered to children with fevers of $102^{\circ} \mathrm{F}$ or lower were as effective as a $10 \mathrm{mg} / \mathrm{kg}$ dose of acetaminophen. In the trials in children with juvenile arthritis, ibuprofen at doses of $30 \mathrm{mg} / \mathrm{kg} /$ day to $40 \mathrm{mg} / \mathrm{kg} /$ day were comparable in efficacy with aspirin at doses of $60 \mathrm{mg} / \mathrm{kg} /$ day to 80 $\mathrm{mg} / \mathrm{kg} /$ day.

\section{Eminase cleared for treatment of myocardial infarction}

The FDA approved Eminase (anistreplase, APSAC) (SmithKline Beecham Philadelphia) as a thrombolytic agent that can be administered to patients with myocardial infarction (MI) over a period of $5 \mathrm{~min}$ utes or less by use of a simple syringe.

Because of its efficacy, Eminase may increase a patient's chance of surviving an MI. In a major mortality study of more than 1000 patients (Lancet $1988 ; 1: 545-549)$, there was a $47 \%$ decrease in mortality at 30 days in patients receiving Eminase as compared with those given a placebo. At 1 year, reduction in mortality was retained at $38 \%$.

The most common complication is bleeding. Eminase demonstrated an overall incidence of $14.6 \%$ in clinical trials, with $5.7 \%$ from puncture sites. In a double-blind, placebo-controlled study, major bleeding events were rare
(1.6\%) in patients receiving the drug compared with patients who received placebo $(1.8 \%)$. Hypotension was reported in $10 \%$. Anaphylactoid reactions have been observed rarely $(0.2 \%)$.

If serious bleeding does occur, concomitant heparin, if used, should be terminated immediately. The administration of protamine should be considered to reverse heparinization. A bleeding tendency can be reversed with appropriate replacement therapy. 